\title{
Effective Interaction of Charged Platelets in Aqueous Solution: Investigations of Colloid Laponite Suspensions by Static Light Scattering and Small-Angle X-Ray Scattering
}

\author{
Li Li ${ }^{a}$, L. Harnau ${ }^{b}$, S. Rosenfeldt ${ }^{a}$, and M. Ballauffo ${ }^{*}$ \\ ${ }^{a}$ Physikalische Chemie I, University of Bayreuth, 95440 Bayreuth, Germany \\ ${ }^{b}$ Max-Planck-Institut für Metallforschung, Heisenbergstr. 3, D-70569 Stuttgart, Germany, \\ and Institut für Theoretische und Angewandte Physik, \\ Universität Stuttgart, Pfaffenwaldring 57, D-70569 Stuttgart, Germany
}

(Dated: June 9, 2021)

\begin{abstract}
We study dilute aqueous solutions of charged disk-like mineral particles (Laponite) by a combination of static light scattering (SLS) and small-angle x-ray scattering (SAXS). Laponite solutions are known to form gels above a certain critical concentration that must be described as non-equilibrium states. Here we focus on the investigation by SLS and SAXS at concentrations below gelation $(c<0.016 \mathrm{~g} / \mathrm{L})$ and at low concentrations of added salt $(0.001$ and $0.005 \mathrm{mM})$. Thus, we have obtained the scattering function of single Laponite platelets as well as the structure factor describing their interaction at finite concentration. A detailed analysis of the combined sets of data proves that the solutions are in a well-defined equilibrium state. Moreover, this analysis demonstrates the internal consistency and accuracy of the scattering functions obtained at finite concentrations. We find that Laponite particles interact through an effective pair potential that is attractive on short range but repulsive on longer range. This finding demonstrates that Laponite solutions exhibit only a limited stability at the concentration of added salt used herein. Raising the ionic strength to $0.005 \mathrm{mM}$ already leads to slow flocculation as is evidenced from the enhanced scattering intensity at smallest scattering angles. All data strongly suggest that the gelation occurring at higher concentration is related to aggregation.
\end{abstract}

PACS numbers: 61.10.-i, 61.25.-f, 64.70.Ja

\section{INTRODUCTION}

Laponite consists of disk-like clay particles with a thickness $L \approx 1 \mathrm{~nm}$ and a radius $R \approx 12.5 \mathrm{~nm}$. Each platelet carries a few hundred elementary charges. For a long time, aqueous suspensions of Laponite have been extensively investigated as a model system for disk-like colloids 1, 2, 3, 4]. A theoretical description of such a well-characterized model clay appeared feasible. Early attempts focussed on the structure of the electric double layer around a single platelet [5, 6, 7], or around two parallel platelets [8]. Much of the recent work was initiated by the theoretical treatment by Hansen and coworkers [9]. In the model of Hansen et al. Laponite platelets and their associated electric double layers are represented by nonintersecting discs carrying a constant electrostatic quadrupole moment. The spatial arrangement of these objects has been investigated by Monte Carlo simulations. The results of these studies point to a reversible sol-gel transition at platelet number density $n>0.06 R^{-3}$ (corresponding to a platelet concentration of $3.4 \%$ by weight), where the structure of the gel phase is strongly reminiscent of a "house-of-cards" [10]. However, the quadrupolar disc model has turned out to be an oversimplification. In particular it is inadequate at very short range, where any multipolar expansion breaks

*E-mail: Matthias.Ballauff@uni-bayreuth.de down, and at long range, where electrostatic interactions between the platelets are exponentially screened.

A screened electrostatic potential between two arbitrary oriented, charged platelets has been worked out within the framework of a linearized Poisson-Boltzmann theory 11, 12, 13, 14]. At a fixed center-to-center distance, this electrostatic potential (always repulsive) is maximized for co-planar platelets, which corresponds to the maximum overlap of electric double layers, and minimized when the platelets are co-axial and parallel. An intermediate potential is found for T-shape perpendicular platelets. At present only preliminary results of Monte Carlo simulations for platelets interacting through such an anisotropic potential are available, indicating that the platelet fluid is in the isotropic phase for platelet number density $n<0.1 R^{-3}[13]$.

Later an interaction site model of Laponite was put forward, and investigated by molecular-dynamics simulations [15] and integral equations [16]. In this model each platelet carries discrete charged sites rigidly arranged on an array inscribed in the disc. Sites on different platelets interact via a repulsive screened Coulomb interaction. Upon varying the platelet number density and screening length, a sol, gel, and crystal phase have been identified. Additional rim charges of opposite sign have been found to lead to T-shaped pair configurations and clustering of the platelets. It should be noted, however, that these models treat the Laponite particles as uniform.

Summing up, several mechanisms have been proposed as origin for the gel formation in Laponite solutions by 
now: First of all, the classical answer to this problem is the formation of a "house of cards"-structure by attractive interactions between the platelets, possibly caused by positive charges on the rims 10]. This includes more recent approaches taking into account the charge distribution around the platelets [16].

As an alternative to this modeling, it was argued that this type of aggregation will only take place at higher ionic strength $(>0.01 \mathrm{mM})$ and may be ruled out as cause for gelation. Instead, the repulsive interaction between the charged platelets leads to a glassy state 4, 17, 18, 19, 20, 21, 22, 23, 24, 25, 26]. Slow timedependent processes within the gel were interpreted in terms of an aging of the glass. Very recently, it has been hypothesized that Laponite suspensions behave like an attractive glass 27, 28]. A comprehensive discussion of the treatment of possible glassy states in Laponite suspensions including a comparison with experimental data was recently given by Mongondry et al. [29].

A markedly different model recently proposed by Nicolai et al. invokes aggregation in order to explain the process of gelation [30, 31, 32]. Weak attraction may lead to a slow aggregation of the particles even at low ionic strength. As a consequence of this, no well-defined phases or phase transitions can be identified anymore. The observations made by Nicolai et al. 29] are in agreement with a study of Martin et al. 33]. Hence, the origins of the sol-gel transition have not yet been fully clarified despite of more than two decades of research.

Most of the theoretical expositions on gelation and structure formation in Laponite suspensions have assumed a system of monodisperse platelets, i.e., thin disks with a radius of $12.5 \mathrm{~nm}$ and a thickness of $1 \mathrm{~nm}$. However, it has become possible to visualize directly the Laponite particles by various techniques as AFM or electron microscopy. From these micrographs it is obvious that Laponite exhibits a considerable polydispersity [34, 35, 36, 37]. A second point that is common to many experimental study is the analysis of obvious non-equilibrium states reached after passing the gel line. As the matter of fact, considerable efforts have been devoted to the investigation of the exact position of the gel line. The experimental difficulties of the investigations of such time-dependent states are at hand. Moreover, no clear information on the nature of the interparticle potential can be derived from essentially non-equilibrium states. An advanced understanding of Laponite suspensions, however, requires a clear delineation of the range of repulsion together with a clear assessment of a possible attractive part of the potential that may come into play at short distances 10].

In this paper we present a comprehensive study of Laponite suspensions in the dilute regime by static light scattering (SLS) and small-angle x-ray scattering (SAXS). We purposely avoid higher concentrations leading to gelled states. Instead, particular care is devoted to the proof that we do look at equilibrium states indeed. Hence, data are taken at different small concentrations of the solute and extrapolated to infinite dilution. This leads to the scattering intensity $I_{0}(q)$ of single platelets as the function of $q$, the magnitude of the scattering vector $\vec{q}(q=(4 \pi / \lambda) \sin (\theta / 2) ; \lambda$ : wavelength of radiation; $\theta$ : scattering angle). $I_{0}(q)$ in turn can be modelled to give the polydispersity. The scattering intensity $I(q)$ obtained at finite but small concentrations is used to determine the "measured structure factor" $S_{M}(q)$ which is related to the pair correlation function $g(r)$ and the interaction potential $V(r)$. However, the appreciable polydispersity of the Laponite particles precludes a direct analysis of $S_{M}(q)$ to yield $V(r)$. Here we shall address this problem within the framework of the "Polymer reference interaction site model" (PRISM). Using the approach developed previously for suspensions of bidisperse platelets [38], size polydispersity of the platelets will be taken into account by a multicomponent PRISM integral equation theory. We shall demonstrate that the combination of precise scattering data with the multicomponent PRISM theory leads to an unambiguous assessment of the interaction potential in solution.

The paper is organized as follows: After the section Experimental we shall delineate in section III the theory of light scattering of dilute suspension. In particular, the check of the measured data for consistency is given. This section contains also the description of the multicomponent interaction site model used for calculating structure factors. Section IV will give describe the comparison with measured scattering data and the resulting effective interaction potential. A final brief section $\mathrm{V}$ will conclude this paper.

\section{EXPERIMENTAL}

\section{A. Materials}

Laponite RD was obtained from Laporte Industries Ltd. and is used for our study without further purification. Laponite powder was added to a $10^{-3} \mathrm{~mol} / \mathrm{l} \mathrm{NaCl}$ solution in distilled water and mixed at high speed with a magnet stirrer for 4 hours. The dispersions were initially turbid, but became clear in 15-20 minutes, indicating that the particles were fully hydrated. All Laponite samples (volume fraction ranging from $0.02 \%$ to $0.16 \%$ ) were prepared separately, without using a stock solution. Vigorous stirring of the solutions was found to be necessary for dispersing particles homogeneously. SAXSexperiments demonstrated that incomplete dissolution leads to the formation of clusters and large aggregates.

The $\mathrm{pH}$ values of all the samples were adjusted to 10 by using $\mathrm{NaOH}$ in order to avoid degradation or dissolution of Laponite particles. Dispersions were kept still overnight before any use in order to achieve equilibrated state. For SAXS experiments the samples were measured without further treatment, as no difference was observed between the scattering intensities of unfiltered dispersion and filtered one by using $0.22 \mathrm{~mm}$ filters (Millipore). For 
static light scattering experiments the dispersions were filtered by using $0.22 \mathrm{~mm}$ filters (Millipore) into quartz cuvettes to eliminate dust and remaining aggregates.

\section{B. Methods}

The scattering intensity $I(q)$ was obtained by static light scattering in the region of smallest $q$-values and by SAXS for higher $q$-values. Static light scattering were performed by a Sofica SLS-Spectrophotometer, using vertically polarized light with a wavelength of $632.8 \mathrm{~nm}$. The sample is contained in a cylindrical $10 \mathrm{~mm}$ diameter quartz cuvette, which is immersed in an index-matching toluene bath of $80 \mathrm{~mm}$ diameter. Scattered light is detected by a photomultiplier, which is mounted on a goniometer arm. The angle range is $35-125$ deg., corresponding to q range of $7.9 \times 10^{-3}-2.3 \times 10^{-2} \mathrm{~nm}^{-1}$.

For SAXS experiments, the data are composed by two sets in different $q$ ranges. One set of data in relatively lower q range were measured at the European Synchrotron Radiation Facility (ESRF) in Grenoble on the high brilliance beam line ID2. The instrumental setup for Laponite samples is as following: wavelength $\lambda=$ $0.0995 \mathrm{~nm}$ with energy $12460 \mathrm{eV}$, sample-detector distance $d=10 \mathrm{~m}$ which covers an experimental range in $q$ between $2.15 \times 10^{-2}$ to $0.67 \times 10^{-1} \mathrm{~nm}^{-1}$. A two-dimensional image-intensified charge-coupled device (CCD) is employed as the detector, which allows the study of the (an)isotropy of the scattering. The other set of SAXS data in q range from 0.07 to $6 \mathrm{~nm}^{-1}$ were obtained in our lab by a Kratky commercial camera. The cameras and the data treatment have been described elsewhere [39].

Density measurements were done in dilute solutions using a DMA 60 apparatus supplied by Paar, Graz, Austria. This gave a mass density of $2.42 \pm 0.12 \mathrm{~g} / \mathrm{cm}^{3}$ for the dissolved Laponite particles. The refractive index increment was measured using the Refraktometer alpha-Ref Typ 1 apparatus supplied by SLS-Systemtechnik (Freiburg, Germany) operating at the wavelength of the apparatus using for static light scattering (632.8 nm).

\section{THEORY}

\section{A. Analysis of particle interaction by light and small-angle $x$-ray scattering}

We consider a dilute isotropic suspension of $N$ monodisperse platelets per volume $V$. The scattering intensity $I(q)$ can be rendered as the product of the intensity $I_{0}(q)$ of single platelets and the structure factor $S(q)$ through $40,41,42]$

$$
I(q)=\frac{N}{V} I_{0}(q) S(q) .
$$

The scattering intensity of a single platelet $I_{0}(q)$ may be used to define the form factor $P(q)$ that is normalized to unity at $q=0$ by

$$
I_{0}(q)=V_{p}^{2}\left(\bar{\rho}-\rho_{m}\right)^{2} P(q),
$$

where $V_{p}$ denotes the volume of the particles, $\bar{\rho}$ is the scattering length density of the particles and $\rho_{m}$ is the respective quantity of the solvent (see the discussion of this point in Ref. [43, 44]). Since the volume fraction is given by $\phi=(N / V) V_{p}$ it follows that $I(q=0) / \phi=$ $V_{p}\left(\bar{\rho}-\rho_{m}\right)^{2}$ where $\bar{\rho}-\rho_{m}$ is the contrast of the particles in the respective solvent. If the concentrations of the solute is expressed through the weight concentrations $c$, $V_{p}$ must be replaced by the molecular weight $M$, that is

$$
I(q)=K c M P(q) S(q) .
$$

In case of SAXS the constant $K$ then follows from the number of scattering units per unit mass of the solute. For light scattering, $K$ is given by 45

$$
K=\frac{4 \pi^{2} \tilde{n}_{0}^{2}\left(\frac{d \tilde{n}}{d c}\right)^{2}}{\lambda^{4} N_{A}},
$$

where $\tilde{n}_{0}$ is the refractive index of the solvent, $(d \tilde{n} / d c)$ is the refractive index increment, $\lambda$ denotes the wavelength of the light, and $N_{A}$ is Avogadro's number.

With complete generality, $P(q)$ may be expanded for small $q$ to give 40,42 ]

$$
P(q) \approx \exp \left(\frac{-R_{g}^{2} q^{2}}{3}\right),
$$

where $R_{g}$ is the radius of gyration of the particles. $I_{0}(q)$ may hence be extrapolated to $q=0$. In general, a platelet can be modelled as a circular disc of radius $R$ and thickness $L$. For randomly oriented monodisperse platelets we have [40]

$$
P(q)=\int_{0}^{\frac{\pi}{2}} d \vartheta \sin \vartheta\left[\frac{4 \sin (q L / 2 \cos \vartheta) J_{1}(q R \sin \vartheta)}{q^{2} R L \cos \vartheta \sin \vartheta}\right]^{2},
$$

where $J_{1}$ denotes the cylindrical Bessel function of first order.

\section{B. Interaction in dilute solution}

The dependence of $S(q)$ on concentration may be expressed in the usual virial series by $40,41,42]$

$$
\frac{1}{S(q)}=1+2 B_{\mathrm{app}} \phi+3 C_{\mathrm{app}} \phi^{2}+\ldots
$$

where the apparent virial coefficient $B_{\text {app }}$ depends on $q$ through 40, 43, 44]

$$
B_{\mathrm{app}}=4 \frac{(\pi / 6) d_{\text {eff }}^{3}}{V_{p}}\left(1-\frac{1}{10} d_{e f f}^{2} q^{2}+\ldots\right),
$$


and $d_{\text {eff }}$ is a measure for an effective diameter of interaction in the dilute regime. The quantity $C_{\text {app }}$ is the correction related to the third virial coefficient. Eq. (8) can be derived easily from the fact that binary interaction prevails if the concentration is low enough. Therefore the pair correlation function $g(r)$ follows simply by $g(r)=\exp [-V(r) / k T]$ [46].The scattering data obtained in the dilute regime can therefore be extrapolated to vanishing concentration by plots of $\phi / I(q)$ against concentration. Moreover, Eq. (8) suggests that the slope of these plots that give the apparent virial coefficient $B_{a p p}$ will diminish with increasing value of $q$ [47]. Hence, a precise analysis of the scattering intensity $I(q)$ obtained for different concentrations leads not only to a proper extrapolation to vanishing volume fraction, but also to parameters characterizing the interaction of the particles: Evidently, Eq. (8) refers only to equilibrium states. The analysis of $I(q)$ obtained in the dilute regime may therefore yield to criteria that allow us to exclude nonequilibrium states.

Arguments derived from first principles demonstrate that $S(q)$ can be expanded into a series of powers in $q^{2}$ [43, 48, 49, 50]

$$
S(q)=S(0)+\alpha q^{2}+\ldots,
$$

where the coefficient $\alpha$ is related to the second moment of the interaction potential $V(r)$ and provides a measure for the range of interaction of the particles in suspension (see the discussion of this point in Ref. [50]). In contrast to Eq. (8), this relation holds for all possible volume fractions. Therefore Eq. (9) provides another check for the data. In particular, it is highly useful to exclude scattering intensities that have been afflicted by the presence of aggregates [43].

\section{Polydispersity}

As mentioned above, previous work has shown that Laponite particles are not monodisperse. The effect of polydispersity must therefore be taken into account and the intensity becomes an appropriate average over all species $i$

$$
I(q)=K \frac{\sum_{i=1}^{s} c_{i} M_{i} P_{i}(q)}{\sum_{i=1}^{s} c_{i} M_{i}} S_{M}(q)=K c M_{w} P_{w}(q) S_{M}(q),
$$

where $P_{i}(q)$ is the normalized form factor of a platelet of species $i$ [Eq. [6] ], $S_{M}$ is the "measured structure factor", and $s$ is the total number of species. Moreover, we have $\sum_{i=1}^{s} c_{i}=c$. The weight-average molecular weight $M_{w}$ of the platelets follows from this as

$$
M_{w}=\frac{\sum_{i=1}^{s} c_{i} M_{i}}{\sum_{i=1}^{s} c_{i}} .
$$

The normalized form factor of the multicomponent system $P_{w}(q)$ can be expressed through the form factors $P_{i}(q)$ of platelets of species $i$ by

$$
P_{w}(q)=\frac{\sum_{i=1}^{s} c_{i} P_{i}(q)}{\sum_{i=1}^{s} c_{i}},
$$

again with $P_{w}(0)=1$. Obviously, all quantities discussed in the previous section as e.g. $B_{a p p}$ and $d_{\text {eff }}$ must be replaced by appropriate mean values.

\section{Multicomponent interaction site model}

A quantitative understanding of correlations and interactions between various colloidal species can be achieved using the well-established techniques of liquid-state theory [46]. The PRISM ("polymer reference interaction site model") theory, originally designed for the study of polymer solutions and melts [51], has been recently extended to investigate various charged and uncharged colloidal systems [16, 52, 53, 54, 55, 56, 57, 58], and mixtures of neutral spherical colloids and polymers [59, 60, 61, 62, 63], as well as like- and oppositely-charged colloids and polyelectrolytes [38, 64]. The key theoretical problem to be addressed here is that of an interpretation of experimental scattering data on very dilute Laponite clay suspensions within the framework of PRISM. The main difference of this work from the previous theoretical studies on monodisperse [16] and bidisperse 38] platelet suspensions is that we take into account polydispersity in the size of the platelets by using a multicomponent PRISM integral equation theory. We consider a multicomponent system involving $s$ species of charged platelets with number densities $n_{i}$, where $1 \leq i \leq s$. Each platelet of species $i$ contains $N_{i}$ equivalent interaction sites. The interaction potential between sites on particles of species $i$ and $j$, carrying the charges $z_{i} e$ and $z_{j} e$, will be of the generic form:

$$
u_{i j}(r)=\frac{z_{i} z_{j} e^{2}}{\varepsilon r} \exp \left(-\kappa_{D} r\right),
$$

where $\lambda_{D}=\kappa_{D}^{-1}$ is the usual Debye screening length. The system under investigation is an aqueous dispersions, but in view of the mesoscopic scale of the platelets, the solvent is modelled as a structureless dielectric continuum providing a macroscopic permittivity $\varepsilon$. Any microscopic counterions or ions from added electrolyte will be considered at the linear response (or Debye-Hückel) level, i.e., they will screen the electrostatic potential due to the interaction sites on the charged platelets on a scale given by the Debye screening length. The underlying BornOppenheimer-like assumption entails that the charge distribution on the mesoscopic particles does not contribute to screening. 


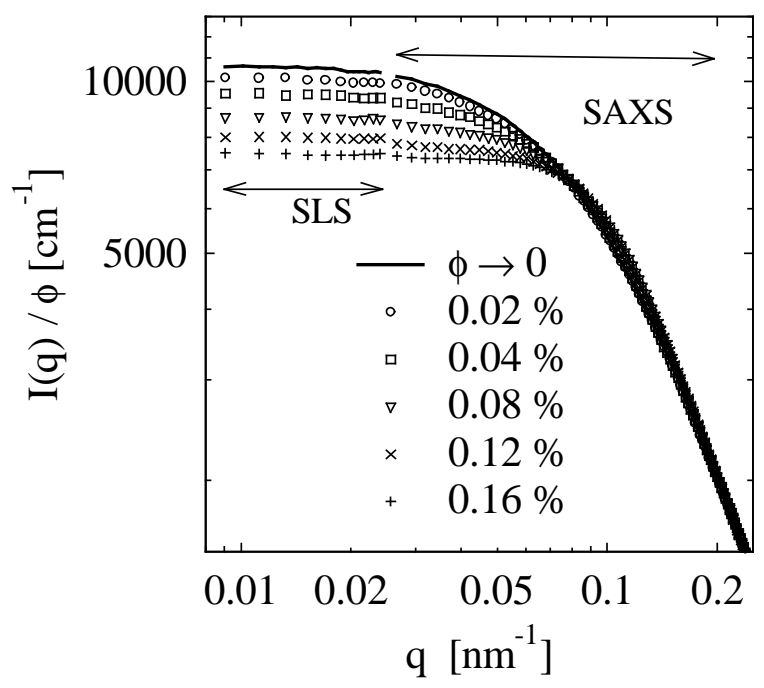

FIG. 1: Absolute scattering intensities of Laponite suspensions normalized to their volume fraction $\phi$ indicated in the graph. The data have been obtained by static light scattering in the region of smallest $q$-values $\left(7.9 \times 10^{-3}-2.3 \times 10^{-2}\right.$ $\left.\mathrm{nm}^{-1}\right)$. Data at higher $q$ were taken by using the beamline ID2 $\left(2.15 \times 10^{-2}\right.$ to $\left.0.67 \times 10^{-1} \mathrm{~nm}^{-1}\right)$ and by a conventional Kratky-camera $\left(0.07\right.$ to $\left.6 \mathrm{~nm}^{-1}\right)$. The solid line refers to the intensity extrapolated to vanishing concentration.

PRISM is based on the assumption that all direct correlation functions between sites on pairs of different platelets are identical. This leaves a total of $s(s+1) / 2$ independent direct correlation functions $c_{i j}(q)$. Corresponding total correlation functions $h_{i j}(q)$ are defined by averaging over the $N_{i} N_{j}$ correlation functions between all pairs of sites $i$ and $j$ on two platelets of the same or different species. The two sets of correlation functions are related by the generalized Ornstein-Zernike equations of the PRISM, which in Fourier space read 51]

$$
h_{i j}(q)=\omega_{i}(q) c_{i j}(q) \omega_{i}(q)+\omega_{i}(q) \sum_{l=1}^{s} c_{i l}(q) n_{l} N_{l} h_{l j}(q),
$$

where $N_{i} n_{i}$ is the number density of interaction sites of platelets of species $i$ and $\omega_{i}(q)=N_{i} P_{i}(q)$. The set of $s(s+1) / 2$ independent Ornstein-Zernike relations must be supplemented by as many closure relations between each pair of total and direct correlation functions. Here we adopt the Laria-Wu-Chandler closure 65] which has recently been used for charged polymers and colloids 16 , 52, 53, 54, 56, 57]

$$
h_{i j}(r)=\ln \left[h_{i j}(r)+1\right]+\omega_{i} \star\left[c_{i j}+\left(k_{B} T\right)^{-1} u_{i j}\right] * \omega_{j},
$$

where the asterics $\star$ denotes a convolution product. In addition, steric effects are taken into account using the Percus-Yevick approximation [46].

The closed set of equations (14) and (15) are solved numerically by a standard iterative procedure to obtain

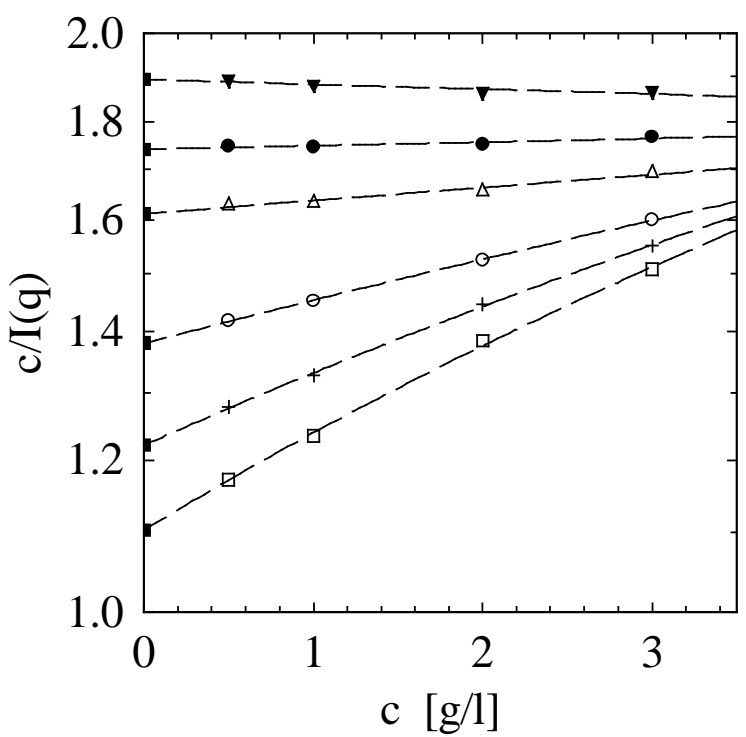

FIG. 2: Extrapolation of the measured scattering intensities $I(q)$ to vanishing concentration according to Eq. (7) and (8). Parameter of the curves is the magnitude $q$ of the scattering vector. The respective values are (in $\mathrm{nm}^{-1}$ ): quadrangles: 0.0215; crosses: 0.037; open circles: 0.0526; open triangles: 0.0681; filled circles: 0.0759; filled trangles: 0.0837 .

partial structure factors

$$
S_{i j}(q)=n_{i} N_{i} \omega_{i}(q)+n_{i} n_{j} N_{i} N_{j} h_{i j}(q) .
$$

Scattering experiments measure the intensity of scattered radiation as a function of momentum transfer. To a multiplicative constant this quantity is the same as the sum of the partial structure factors $\sum_{i, j=1}^{s} S_{i j}(q)$ which is denoted as the static structure factor. Most experimental papers on polyectrolytes, however, report the ratio of the static structure factor to the form factor. Therefore we compare in the following section the theoretical prediction for the function

$$
S_{M}(q)=\frac{\sum_{i, j=1}^{s} S_{i j}(q)}{\sum_{i=1}^{s} n_{i} N_{i} \omega_{i}(q)}
$$

to experimental data. In keeping with the experimental terminology we refer to $S_{M}(q)$ as the measured structure factor.

\section{RESULTS AND DISCUSSION}

\section{A. Static scattering experiments: consistency check}

As mentioned in the Introduction, the present investigation focuses on the dilute regime in which no gelation 


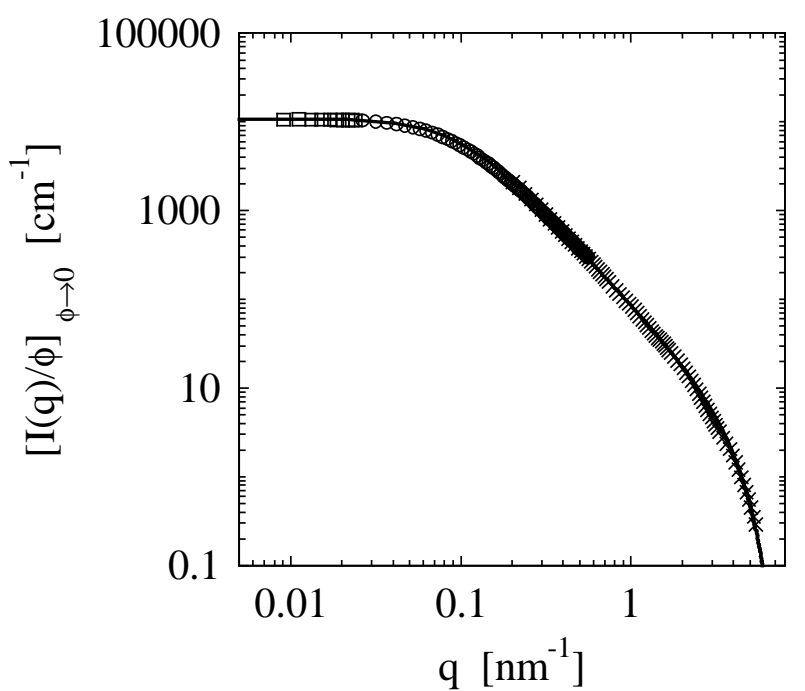

FIG. 3: Intensity $I_{0}(q) / \phi$ extrapolated to vanishing volume fraction $\phi$. The data have been obtained by static light scattering in the region of smallest $q$-values $\left(7.9 \times 10^{-3}-2.3 \times 10^{-2}\right.$ $\left.\mathrm{nm}^{-1}\right)$. Data at higher $q$ were taken by using the beamline ID2 $\left(2.15 \times 10^{-2}\right.$ to $\left.0.67 \times 10^{-1} \mathrm{~nm}^{-1}\right)$ and by a conventional Kratky-camera $\left(0.07\right.$ to $\left.6 \mathrm{~nm}^{-1}\right)$. The solid line marks the fit according to Eq. (6).

occurs. However, no concentration is small enough to disregard the influence of mutual interaction of the platelets, i.e., of $S(q)$. Moreover, the discussion in section $\amalg$ has demonstrated that scattering data in this regime furnish valuable data as $B_{a p p}$ that may be used for a consistency check. As a first step in this analysis scattering data are therefore taken at small concentration $(\phi \leq 0.16 \%)$ and carefully extrapolated to vanishing concentration. Since we aim at the limits for vanishing scattering angles for all quantities under consideration, the $q$-range accessible by SAXS was not sufficient. Hence, static light scattering was used to obtained data at smallest $q$-values possible in order to supplement the analysis by synchrotron SAXS. For the highest scattering angles the data obtained by synchrotron SAXS were supplemented by intensities taken by a conventional Kratky-camera. This device can take data up to $6 \mathrm{~nm}^{-1}$.

In all cases absolute scattering intensities have been obtained. For light scattering, the constant $K$ in Eq. (4) was found to $K=(4.99 \pm 1) 10^{-8} \mathrm{~cm}^{2} \mathrm{Mol} / \mathrm{g}^{2}$ from the measured refractive increment $(d \tilde{n} / d c=0.083 \pm 0.008$ $\mathrm{mL} / \mathrm{g}$ ). In case of SAXS the respective optical constant $K$ in Eq. (33) was found to be $K=0.0445 \mathrm{Mol} / \mathrm{g}^{2}$. All absolute intensities agreed within the present limits of error $( \pm 5 \%)$.

Previous investigations by Bonn et al. 22 and by Nicolai and Cocard [66] have shown that even dilute aqueous suspensions of Laponite may contain aggregates that must be filtered off, in particular for light scattering experiments. Hence, all suspensions have been carefully filtrated prior to use. Moreover, special care was exerted

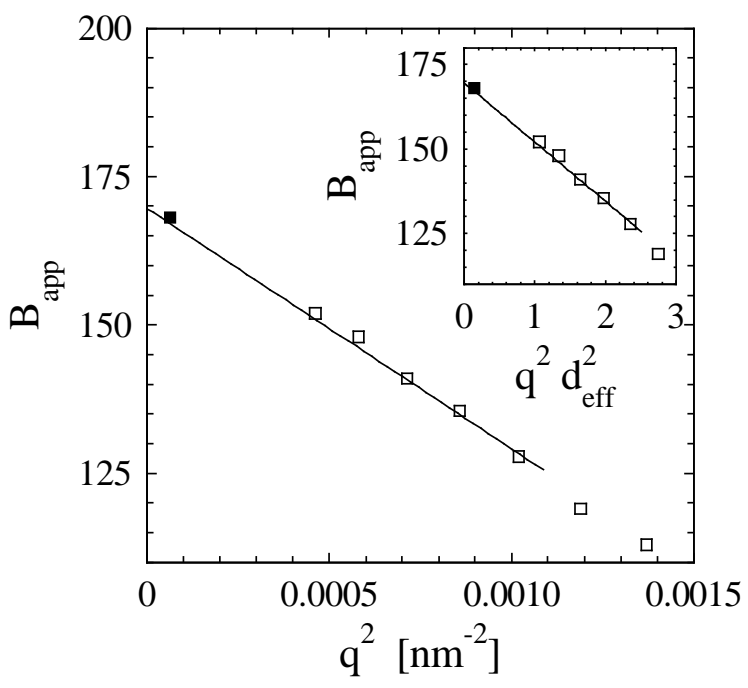

FIG. 4: Apparent second virial coefficient $B_{a p p}$ (cf. Eq. (8)) of Laponite suspensions in the dilute regime. the filled circle denotes the value obtained by static light scattering whereas the other points have been obtained by SAXS. The effective diameter $d_{e f f}$ deriving from this fit results to $48 \mathrm{~nm}$.

in preparing the solutions by carefully stirring for a prolonged time. If the time for the process of dissolution was chosen to be short or without vigorous stirring, aggregates resulted that showed up in the region of smallest angles. Evidently, the pH 10 and the ionic strength are further parameters of central importance. Most of the data to be discussed here were taken from solutions in which the ionic strength was set to $0.001 \mathrm{mM}$ by $\mathrm{NaCl}$.

Figure [1 displays the data obtained at low ionic strength for various volume fractions $\phi$ calculated from the weight concentrations of the solute and its density in solution $\left(2.42 \pm 0.12 \mathrm{~g} / \mathrm{cm}^{3}\right)$. First of all, the data obtained from SLS fit well together with the data obtained from SAXS. Moreover, the influence of the structure factor $S(q)$ cannot be disregarded even at the lowest volume fraction. This is due to the electrostatic stabilization that must lead to an effective diameter of interaction $d_{\text {eff }}$ which is larger than the largest dimension of the platelets. Figure 2 demonstrates that these data can be safely extrapolated to vanishing concentration by use of Eq. (7) and (8). Plots of $c / I(q)$ vs. $c$ lead to straight lines. The resulting intercepts are given as solid lines in Fig. 1] Even at the smallest concentration $(\phi=0.02 \%)$ the scattering intensities deviate from the value resulting from this extrapolation. Only at higher scattering angles the influence of mutual interaction is absent in full accord with Eq. (8).

Figure 3 displays the intensity extrapolated to vanishing concentration for the entire $q$-range available from the combination of three devices, namely static light scattering, synchrotron radiation, and a conventional Kratkycamera. The rather flat region of small angles is due to the fact that $I_{0}(q)$ must follow Guinier's law (see 


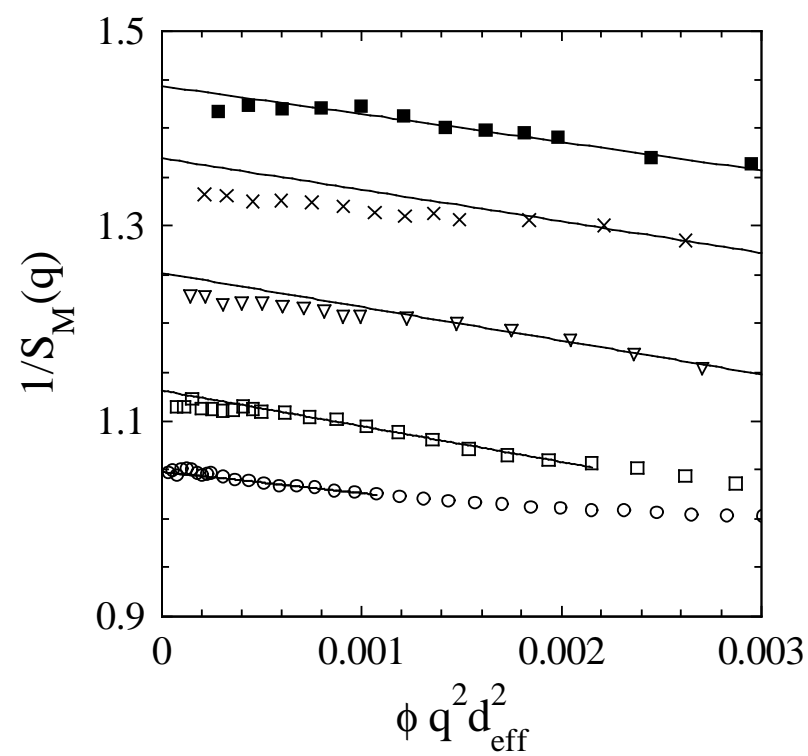

FIG. 5: Consistency check of the structure factor $S_{M}(q)$ in the region of small $q$-values according to Eq. (7) and (8).

Eq. (51)) at small values of $q$. From a Guinier plot of these data (not shown) we obtained a weight average of the platelets $M_{w}=930 \pm 190 \mathrm{~kg} / \mathrm{Mol}$ and a radius of gyration $R_{g}=13.4 \mathrm{~nm}$. At intermediate $q$ the slope of -2 is seen predicted by Eq. (6). Finally, at highest $q$-values the scattering intensity is greatly diminished because of the first minimum of $P(q)$ which is located at $q=7.1$ $\mathrm{nm}^{-1}$.

The form factor $P(q)$ of a thin platelet can be easily calculated according to Eq. (6). From this fit it becomes obvious that polydispersity must be included to give a satisfactory fit. This is due to the fact that Eq. (6) leads to a wavy from of $I_{0}(q)$ for monodisperse platelets which is smoothed out by polydispersity. The solid line shows the resulting fit obtained for a Schulz-Zimm-distribution 67, 68] with a polydispersity expressed through $M_{w} / M_{n}=1.5$. The weight-average radius $R_{w}$ is given by $10.5 \mathrm{~nm}$, the thickness is found to be $0.9 \mathrm{~nm}$, and the calculated weight average [Eq. (11)] is in agreement with the measured value. We reiterate that Laponite platelets exhibit a appreciable polydispersity that can be deduced from this analysis. Obviously, this must be taken into account when modelling the interaction of the particles at finite volume fraction.

The slope of the curves shown in Fig. 2 can now be evaluated to yield the apparent second virial coefficients $B_{a p p}$ defined through Eq. (7). Figure 4 displays the resulting data and a fit according to Eq. (8). ¿From this fit the effective diameter $d_{\text {eff }}$ was obtained to $48 \mathrm{~nm}$ which is considerably higher than the largest dimension of the platelets (see above). This can be traced back to the electrostatic repulsion that is already operative before the platelets touch each other. Hence, $d_{\text {eff }}>2 R_{w}$ and $B_{a p p}$ cannot be calculated from the dimensions of

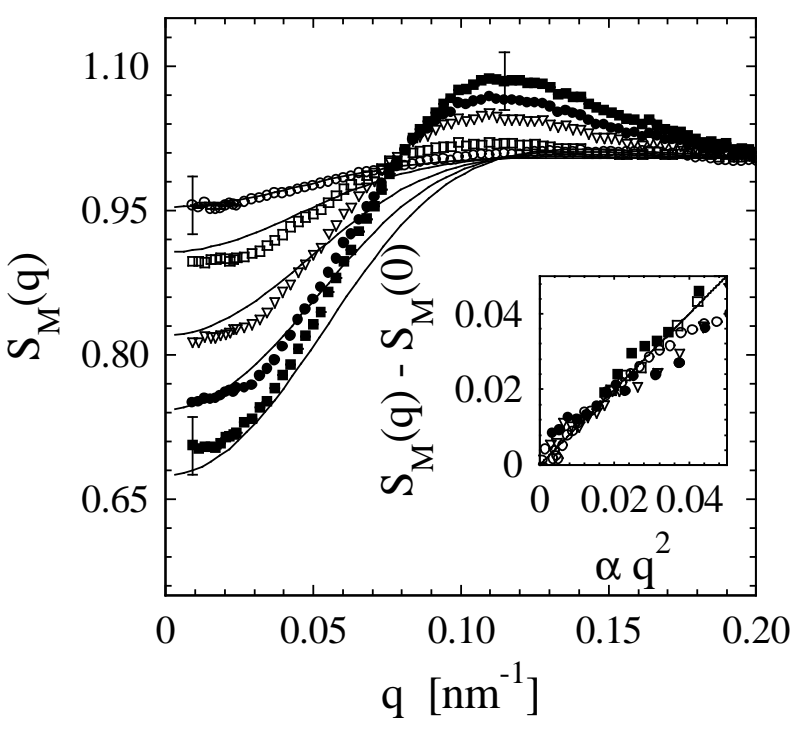

FIG. 6: Structure factor $S_{M}(q)$ determined experimentally for various volume fractions (open circles: $0.02 \%$; open squares: $0.04 \%$; open triangles: $0.08 \%$; solid circles: $0.12 \%$; solid squares: $0.16 \%)$. The solid lines represent the corresponding structure factors computed according to Eq. (17) and assuming a purely repulsive interaction between the platelets [Eq. [13]. The inset shows the consistency check of the experimental data according to Eq. (9).

the platelets in solution. The inset gives the same plot but with the abscissa scaled by $\phi d_{\text {eff }}$ as suggested by Eq. (8). A similar plot is displayed in Fig. [5] showing the extrapolation to vanishing concentration as in Fig. [3] Figure 5 demonstrates that approximately parallel lines result from this scaling of the abscissa. Thus, the data obtained at low concentrations follow Eq. (8). This indicates that these solutions fully qualify as equilibrium states.

The intensity referring to $\phi \longrightarrow 0$ may now be used to determine the structure factor $S(q)$ for nonzero values of $\phi$. The foregoing discussion has clearly revealed, however, that the Laponite particles exhibit an appreciable polydispersity. The structure factor obtained from this division should therefore be treated as a "measured" quantity $S_{M}(q)$ that contains explicitly a contribution of the size distribution. Figure [6] displays the resulting $S_{M}(q)$ obtained for the low ionic strength of 0.001 $\mathrm{mM}$. The inset shows the consistency check according to Eq. (9). It underscores again the fact that we are dealing with equilibrium states. Figure 6 shows that $S_{M}(q)$ is considerably lowered at vanishing scattering angle and exhibit a shallow maximum around $q=0.11 \mathrm{~nm}^{-1}$. As mentioned above, most of the investigations using scattering methods have been conducted near the concentration of gelation, i.e., at much higher concentrations, and the structure factor has not been extracted. Hence, we cannot compare the present data directly with similar measurements from literature. 


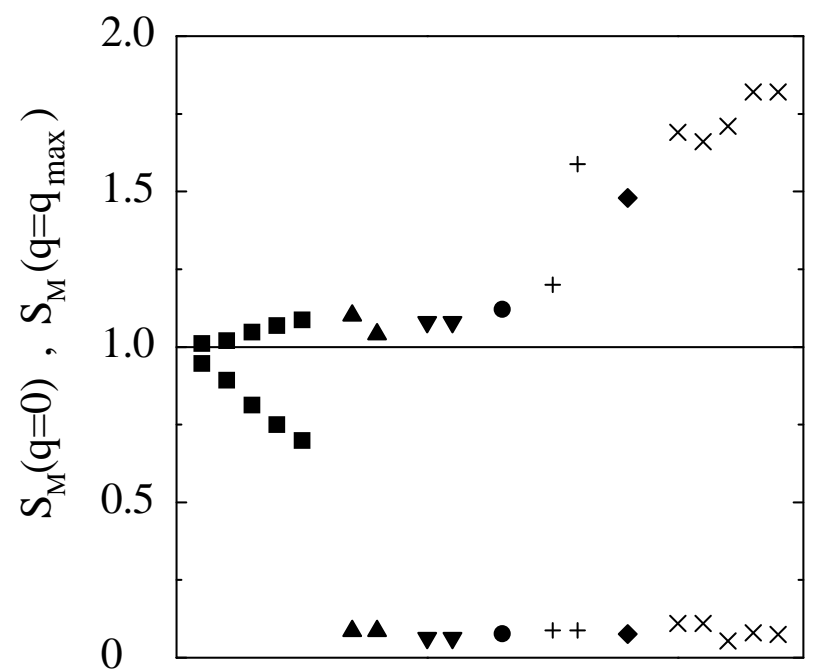

FIG. 7: The structure factor at zero scattering vector $S_{M}(q=0)$ (lower symbols) together with the structure factor at the main peak $S_{M}\left(q=q_{\max }\right)$ (upper symbols) for various suspensions: Laponite at five volume fractions: $0.02 \%$, $0.04 \%, 0.08 \%, 0.12 \%, 0.16 \%$ from left to right (squares); polystyrenesulfonate of length $L=6.8 \mathrm{~nm}$ at two concentrations: $0.1 \mathrm{~mol} / \mathrm{L}$ and $0.2 \mathrm{~mol} / \mathrm{L}$ from left to right (up triangles [83]); polystyrenesulfonate of length $L=40 \mathrm{~nm}$ at two concentrations: $0.1 \mathrm{~mol} / \mathrm{L}$ and $0.2 \mathrm{~mol} / \mathrm{L}$ from left to right (down triangles [83]); DNA of length $L=57 \mathrm{~nm}$ at $0.05 \mathrm{~mol} / \mathrm{L}$ (circles 83]); DNA of length $L=380 \mathrm{~nm}$ at $0.05 \mathrm{~mol} / \mathrm{L}$ (diamonds [83]); proteoglycan at two different salt concentrations: $0.05 \mathrm{mM}$ and $0 \mathrm{mM}$ from left to right (plus-symbols [77]); tobacco mosaic virus at five concentrations: $0.11 \mathrm{mg} / \mathrm{mL}, 0.27$ $\mathrm{mg} / \mathrm{mL}, 0.43 \mathrm{mg} / \mathrm{mL}, 1.05 \mathrm{mg} / \mathrm{mL}, 2.07 \mathrm{mg} / \mathrm{mL}$ from left to right (crosses 84]).

The results for the structure factor as calculated according to Eqs. (13) - (17) are compared to the experimental scattering data in Fig. 6. The adjustable parameter related to the potential of interaction is the effective charge of the platelets which is much smaller than the structural charge of the platelets, due to counterion "condensation" 8, 13, 70]. It is the strong reduction of the bare charge which allows the use of linearized Poisson-Boltzmann theory, leading to the simple exponential Debye screening of the Coulomb interaction [Eq. (13)]. In the calculations we use a fixed surface charge density which is defined as the charge of a platelet of species $i$ divided by the area $A_{i}=\pi R_{i}^{2}$ of the face of the platelet. The calculated structure factors agree with the experimental data for small scattering vectors, while pronounced deviations are visible in the intermediate scattering vector regime. Particularly, the theoretical calculations predict considerably smaller peaks of the structure factor than observed experimentally. Increasing the surface charge density, as compared to the one used in Fig. 6] leads to an increase of the height of the

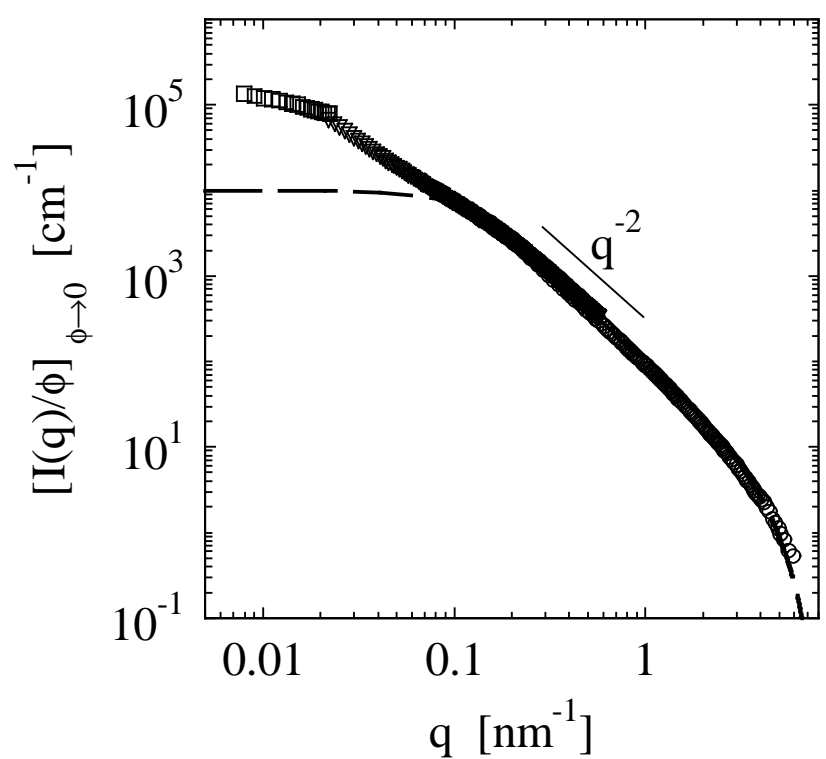

FIG. 8: Slow aggregation of Laponite particles at higher ionic strength $(0.005 \mathrm{mM})$. The intensity $I_{0}(q) / \phi$ was extrapolated to vanishing volume fraction $\phi$ (cf. the discussion of Fig. 3). Note the much higher intensity obtained at small scattering vectors as compared to the case of the lower ionic strength (0.001 mM) in Fig. 3

peaks of the calculated structure factors and to a decrease of the structure factors at small scattering vectors $q \rightarrow 0$. We emphasize that neither using different model parameters (surface charge density, Debye screening length, and size of the platelets) nor using a different distribution function (Gaussian, log-normal, and Tung distribution) lead to an agreement with the experimental data.

Since structure factors and pair correlation functions of polyelectrolyte solutions calculated within the same theoretical framework are in good agreement with experimental data and computer simulations 38, 52, 53, 54, 56], it is worthwhile to compare scattering data for polyelectrolytes with the data obtained for Laponite. Figure 7 displays $S_{M}(q=0)$ which is proportional to the isothermal compressibility together with $S_{M}\left(q=q_{\max }\right)$ for various polyelectrolytes and Laponite, where $q_{\max }$ is the absolute value of the scattering vector at the main peak. The structure factors of the polyelectrolytes exhibit a main peak $S_{M}\left(q=q_{\max }\right)>1$ and a small value $S_{M}(q=0)<0.11$ for small scattering vectors, while the structure factors of Laponite are charcterized by a main peak and a rather large value $0.7<S_{M}(q=0)<0.95$ for small scattering vectors. The enhancement of the small angle $(q \rightarrow 0)$ value of $S_{M}(q)$ of Laponite as compared to the polyelectrolytes signals increased density fluctuations. Since the polyelectrolyte solutions remain liquid-like even at higher concentrations, the observed qualitative different behavior of the structure factors of Laponite may be considered as indicative of the sol-gel transition at higher concentrations. Notice that the val- 
ues of $S_{M}(q=0)$ for the Laponite suspensions under considerations are smaller than 1 and decrease with increasing concentration in contrast to strong small $q$ upturns which have been observed experimentally for clay suspensions in the gel phase [18, 20, 21, 71, 72, 73, 74, 75], polyelectrolyte gels [76] and mixtures [77], polystyrenesulfonate ion exchange resins 78], and low ionic strength polyelectrolyte solutions [79, 80, 81]. These strong upturns signal strong concentration fluctuations indicative of aggregation, or spinodal instability reminiscent of the behaviour observed in recent computer simulations 82 . and PRSIM integral equations [38] of mixtures of oppositely charged particles.

A final point to be discussed at this point is the stability of Laponite suspensions at higher ionic strength. Figure 8 displays the intensity obtained for an ionic strength of $0.005 \mathrm{mM}$. The same procedures have been applied as in case of the data discussed above. The intensities have been extrapolated to vanishing concentration in order to remove all alterations due to $S_{M}(q)$. The dashed line marks the form factor obtained previously for $0.001 \mathrm{mM}$ as described above. It is obvious that the intensity measured at small scattering angles is considerably higher than in case of the lower ionic strength. We interpret this as the onset of a slow coagulation process. Hence, a salt concentration as low as $0.005 \mathrm{mM}$ already suffices to lead to the built-up of aggregates visible in the scattering experiment at small $q$. This points clearly to the limited stability of the Laponite platelets in solutions which will be further discussed below.

\section{B. Effective interaction potential}

The results of the preceding subsection indicate that the disagreement between the experimental and theoretical results may be due the purely repulsive screened Coulomb potential used in the calculations [Eq. (13)]. Recently Ruzicka et al. 85. have suggested that at low concentrations the effective interaction between Laponite platelets are characterized by a competition of long-range Coulomb repulsion and short-range van der Waals attraction similar to colloidal systems and protein solutions [86, 87, 88]. Moreover, the charge density is higher on the face than on the rim of a platelet, which leads to a modification of the interaction potential between sites on particles $i$ and $j$ [Eq. (13)] within the computationally very demanding multicomponent PRISM theory. Here we attempt a mesoscopic coarse graining, whereby particles act via an effective potential $V(r)$. To this end we solve numerically the Ornstein-Zernike equation

$$
h(q)=c(q)+n c(q) h(q)
$$

together with hypernetted-chain closure relation

$$
h(r)=\ln [h(r)+1]+c(r)+\left(k_{B} T\right)^{-1} V(r) .
$$

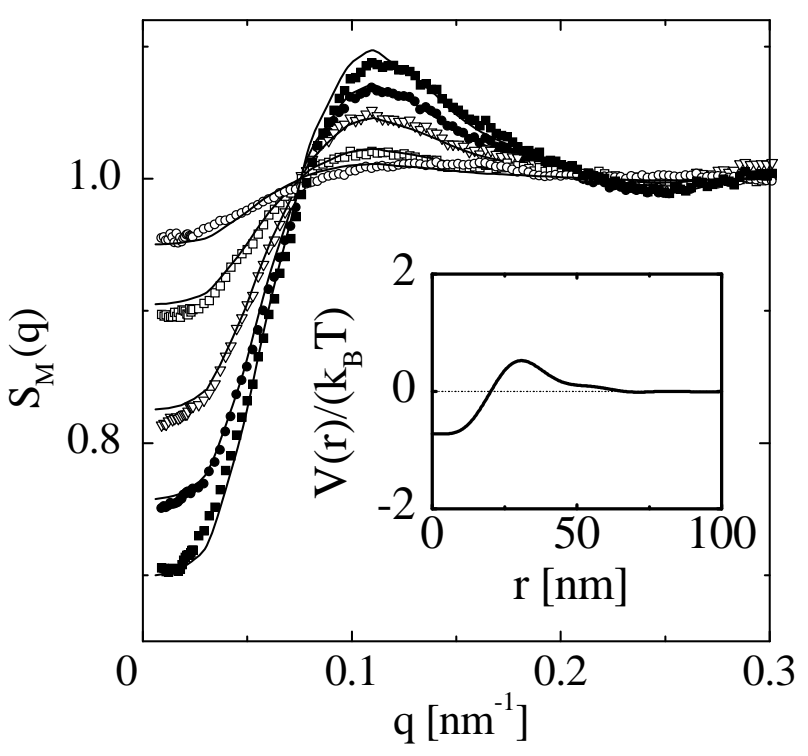

FIG. 9: Structure factor $S_{M}(q)$ determined experimentally for various volume fractions (open circles: $0.02 \%$; open squares: $0.04 \%$; open triangles: $0.08 \%$; solid circles: $0.12 \%$; solid squares: $0.16 \%$ ). The solid lines represent the results of the integral equation theory [Eqs. (18) - 201] using the effective potential $V(r)$ shown in the inset.

These equations follow from Eqs. (14) and (15) for $s=1$, $\omega_{i}(q)=1$, and $N_{i}=1$. The calculated structure factor

$$
S(q)=1+n h(q)
$$

is compared in Fig. (9) with the experimental data. For all volume fraction, good agreement with experiment is achieved with a density-independent effective potential $V(r)$ that is attractive for small distances but repulsive for larger distances (see the inset in Fig. (9)). We emphasize that the effective potential fulfills the stability condition $\int_{0}^{\infty} d r r^{2} V(r)>0$ discussed by Ruelle [89], and provides a representation of the underlying manybody interactions in the system. Thermodynamic properties can be calculated from the compressibility relation $S(q=0)=n k_{B} T \kappa_{T}$, where $\kappa_{T}$ is the isothermal compressibility [46]. The osmotic pressure is evaluated as $P=k_{B} T \int d n S(q=0)^{-1}$. Moreover, the correlation functions obtained from the Ornstein-Zernike equation can be used as input for a Langevin equation for the dynamic structure factor [55] allowing one to study dynamical properties.

Finally, it is instructive to rationalize our results using simple arguments. The repulsion due to the overlap of the counterion diffusive layers can be replaced by a renormalized hard-core repulsion with an effective diameter equal to $2\left(R_{w}+\lambda_{D}\right)=41 \mathrm{~nm}$, where the Debye screening length has been approximated by $\lambda_{D}=\left(8 \pi l_{B} n s\right)^{-1 / 2}$, where $l_{B}=0.714 \mathrm{~nm}$ is the Bjerrum length and $2 n_{s}$ is the particle number density of salt ions at ionic strength $0.001 \mathrm{mM}$. This effective di- 
ameter is somewhat smaller than $d_{e f f}=48 \mathrm{~nm}$ obtained from Eq. (8). Another length of interest is the estimated average distance between two platelets defined as $d_{h}=\left(\phi / V_{w}\right)^{-1 / 3}$, where $V_{w}=\pi R_{w}^{2} L$ is the average particle volume. For $\phi=0.02 \%$ we find $d_{h}=116 \mathrm{~nm}$ and for $\phi=0.16 \%, d_{h}=58 \mathrm{~nm}$. As is apparent from Fig. 9 the correlation length associated with the main peak of the structure factor, $d_{c}=2 \pi / q_{\max } \approx 53 \mathrm{~nm}$ is independent of the volume fraction in contrast to results for Laponite at very low ionic strength (0.0001 M; 74]). The correlation length $d_{c}$ for the samples shown in Fig. 9] is related to the effective diameter $d_{e f f}$ rather than to average distance between two platelets defined as $d_{h}$. However, the fact that $d_{\text {eff }}$ is comparable to $d_{h}$ for $\phi=0.16 \%$ indicates that the sol-gel transition occurs already at a volume fraction slightly larger than $\phi=0.16 \%$ for which $d_{\text {eff }} \approx d_{h}$, in agreement with our experimental findings. The main peak at the scattering vector $q_{\max }$ for gel systems reflects the short interparticle distance between connected particles of effective diameter. The pronounced excess scattering at small values of $q$ is due to correlations associated with the network structure.

\section{CONCLUSION}

A precise analysis of the static scattering intensity of dilute aqueous Laponite solutions has been presented. The combination of static light scattering with smallangle $\mathrm{x}$-ray scattering leads to a wide range of the mag- nitude $q$ of the scattering vector so that reliable data of the structure factor $S_{M}(q)$ could be obtained. Special care was taken to show that the present data refer to true equilibrium states. The form factor obtained from the scattering intensity extrapolated to vanishing volume fraction agrees with the calculated one for polydisperse platelets (see Figure 3). The structure factor $S_{M}(q)$ is characterized by a main Coulomb peak $S_{M}\left(q=q_{\max }\right)>1$ and a value $0.7<S_{M}(q=0)<0.95$ for small scattering vectors corresponding to a rather large isothermal compressibility, in contrast to various polyelectolyte solutions (see Figure [7). The analysis of $S_{M}(q)$ which includes the marked effect of polydispersity demonstrates unambiguously that the Laponite platelets interact via a potential that is attractive on short distances but repulsive on longer distances (see inset of Figure 91. This clearly points to the role of short-range attraction for the process of coagulation at higher volume fractions. Moreover, it explains that higher concentration of added salt lead to aggregation because of the increased screening of the repulsive Coulomb interaction between the Laponite platelets (see Figure 8).

\section{ACKNOWLEDGMENT}

The authors gratefully acknowledge financial support by the Deutsche Forschungsgemeinschaft, Forschergruppe "Peloide". We acknowledge allocation of beamtime by the European Synchrotron Radiation Facility, Grenoble, France.
[1] J. D. F. Ramsay, J. Colloid Interface Sci. 109, 441 (1986).

[2] J. D. F. Ramsay, S. W. Swanton, and J. Bunce, J. Chem. Soc. Faraday Trans. 86, 3919 (1990).

[3] J. D. F. Ramsay and P. Lindner, J. Chem. Soc. Faraday Trans. 89, 4207 (1993).

[4] A, Mourchid, A. Delville, J. Lambard, E. Lecollier, and P. Levitz, Langmuir 11, 1942 (1995).

[5] F. R. C. Chang and G. Sposito, J. Colloid Interface Sci. 163, 19 (1994).

[6] E. Trizac and J.-P. Hansen, Phys. Rev. E 56, 3137 (1997).

[7] J.-P. Hansen and E. Trizac, Physica A 235, 257 (1997).

[8] A. Delville, J. Phys. Chem. B 103, 8296 (1999).

[9] M. Dijkstra, J.-P. Hansen, and P. A. Madden, Phys. Rev. E 55, 3044 (1997).

[10] H. van Olphen, An Introduction to Clay Colloid Chemistry, 2nd. ed., (Wiley, New York, 1977).

[11] J.-P. Hsu and M.-T. Tseng, Langmuir 13, 1810 (1997).

[12] D. G. Rowan, J.-P. Hansen, and E. Trizac, Mol. Phys. 98, 1369 (2000).

[13] R. Agra, E. Trizac, and L. Bocquet, Eur. Phys. J. E. 15, 345 (2004).

[14] E. Trizac, L. Bocquet, R. Agra, J.-J. Weis, and M. Aubouy, J. Phys. Cond. Mat. 14, 9339 (2002).

[15] S. Kutter, J.-P. Hansen, M.Sprik, and E. Boek, J. Chem. Phys. 112, 311 (2000).
[16] L. Harnau, D. Costa, and J.-P. Hansen, Europhys. Lett. 53, 729 (2001).

[17] M. Kroon, G. Wegdam, and R. Sprik, Phys. Rev. E 54, 6541 (1996).

[18] M. Kroon, W. L. Vos, and G. H. Wegdam, Phys. Rev. E 57, 1962 (1998)

[19] A, Mourchid, E. Lecollier, H. van Damme, and P. Levitz, Langmuir 14, 4718 (1998).

[20] A, Mourchid and P. Levitz, Phys. Rev. E 57, R4887 (1998).

[21] D. Bonn, H. Tanaka, G. Wegdam, H. Kellay, and J. Meunier, Europhys. Lett. 45, 52 (1999).

[22] D. Bonn, H. Kellay, H. Tanaka, G. Wegdam, J. Meunier, Langmuir 15, 7534 (1999).

[23] A. Knaebel, M. Bellour, J. P. Munch, V. Viasnoff, F. Lequeux, and J. M. Harden, Europhys. Lett. 52, 73 (2000).

[24] B. Abou, D. Bonn, and J. Meunier, Phys. Rev. E 64, 021510 (2001).

[25] D. Bonn, S. Tanase, B. Abou, H. Tanaka, and J. Meunier, Phys. Rev. Lett. 89, 015701 (2002).

[26] M. Bellour, A. Knaebel, J. L. Harden, F. Lequeux, and J.-P. Munch, Phys. Rev. E 67, 031405 (2003).

[27] H. Tanaka, J. Meunier, and D. Bonn, Phys. Rev. E 69, 031404 (2004), and further citations given there. 
[28] H. Tanaka, S. Jabbari-Farouji, J. Meunier, and D. Bonn, Phys. Rev. E 71, 021402 (2005).

[29] P. Mongondry, J. F. Tassin, and T. Nicolai, J. Colloid Interface Sci. 283, 397 (2005).

[30] T. Nicolai and S. Cocard, Eur. Phys. J. E. 5, 221 (2001).

[31] T. Nicolai and S. Cocard, J. Colloid Interface Sci. 244, 51 (2001).

[32] P. Mongondry, T. Nicolai, and J.-F. Tassin, J. Colloid Interface Sci. 275, 191 (2004).

[33] C. Martin, F. Pignon, J.-M. Piau, A. Magnin, P. Lindner, and B. Cabane, Phys. Rev. E 66, 021401 (2002).

[34] I. Bihannic, L. J. Michot, B. S. Lartiges, D. Vantelon, J. Labille, F. Thomas, J. Susini, M. Salome, and B. Fayard, Langmuir 17, 4144 (2001).

[35] E. Balnois, S. Durand-Vidal, and P. Levitz, Langmuir 19, 6633 (2003).

[36] N. N. Herrera, J.-M. Letoffe, J.-L. Putaux, L. David, and E. Bourgeat-Lami, Langmuir 20, 1564 (2004).

[37] E. S. H. Leach, A. Hopkinson, K. Franklin, and J. S. van Duijneveld, Langmuir 21, 3821 (2005).

[38] L. Harnau and J.-P. Hansen, J. Chem. Phys. 116, 9051 (2002).

[39] N. Dinegnouts, J. Bolze, D. Pötschke, M. Ballauff, Adv. Polym. Sci. 144, 1 (1999).

[40] A. Guinier and G. Fournet, Small-angle scattering of $X$ rays, (Wiley, New York, 1955).

[41] L. A. Feigin and D. I. Svergun, Structure Analysis by Small-Angle X-Ray Scattering and Neutron Scattering, (Plenum Press, New York, 1987).

[42] J. S. Higgins and H. C. Benoît, Polymers and Neutron Scattering, (Clarendon Press, Oxford, 1994).

[43] S. Rosenfeldt, N. Dingenouts, M. Ballauff, P. Lindner, C. N. Likos, N. Werner, and F. Vögtle, Macromol. Chem. Phys. 203, 1995 (2002).

[44] M. Ballauff, in Structure and Dynamics of Polymer and Colloidal Systems, (NATO ASI Series Vol. 568, R. Pecora and R. Borsali, Eds.; Kluwer Academic Publishers, Dordrecht, 2002).

[45] Ch. Tanford, Physical Chemistry of Macromolecules, John Wiley, New York, 1961.

[46] J.-P. Hansen and I. R. McDonald, Theory of Simple Liquids, 2d edition, (Academic Press, London, 1986).

[47] This is in contrast to the well-known Zimm-theory of dilute polymer solutions (see Ref. 42] and further citations given there). In this theory the polymer chain is assumed to be infinitely thin, that is, $D \approx 0$. As a consequence of this, $B_{a p p}$ coincides with its thermodynamic limit for all $q$-values.

[48] A. K. van Helden and A. Vrij, J. Colloid Interface Sci. 78, 312 (1980).

[49] U. Apfel, R. Grunder, and M. Ballauff, Colloid Polym. Sci. 272, 820 (1994)

[50] A, Weiss, K. D. Hörner, and M. Ballauff, J. Colloid Interface Sci. 213, 417 (1999).

[51] For a review, see K. S. Schweizer and J. G. Curro, Adv. Chem. Phys. 98, 1 (1997).

[52] A. Yethiraj and C.-Y. Shew, Phys. Rev. Lett. 77, 3937 (1996).

[53] C.-Y. Shew and A. Yethiraj, J. Chem. Phys. 106, 5706 (1997).

[54] L. Harnau and P. Reineker, J. Chem. Phys. 112, 437 (2000).

[55] L. Harnau, J. Chem. Phys. 115, 1943 (2001).

[56] C.-Y. Shew and A. Yethiraj, J. Chem. Phys. 116, 5308
(2002).

[57] T. Hofmann, R. G. Winkler, and P. Reineker, J. Chem. Phys. 119, 2406 (2003).

[58] L. V. Zherenkova, P. G. Khalatur, and A. R. Khokhlov, J. Chem. Phys. 119, 6959 (2003).

[59] A. Yethiraj, C. Hall, and R. Dickman, J. Colloid Interface Sci. 151102 (1992).

[60] P. G. Khalatur, L. V. Zherenkova, and A. R. Khokhlov, J. Phys. II France 7, 543 (1997).

[61] A. P. Chatterjee and K. S. Schweizer, J. Chem. Phys. 109, 10464 and 10477 (1998).

[62] M. Fuchs, and K. S. Schweizer, Europhys. Lett. 51, 621 (2000).

[63] D. Costa, J.-P. Hansen, and L. Harnau, Molec. Phys. 103, 1917 (2005).

[64] P. G. Ferreira, M. Dymitrowska, and L. Belloni, J. Chem. Phys. 113, 9849 (2000).

[65] D. Laria, D. Wu, and D. Chandler, J. Chem. Phys. 95, 4444 (1991).

[66] T. Nicolai and S. Cocard, Langmuir 16, 8189 (2000).

[67] G. V. Schulz, Z. Phys. Chem. 43, 25 (1939).

[68] B. H. Zimm, J. Chem. Phys. 16, 1099 (1948).

[69] N. J. Wagner, R. Krause, A. R. Rennie, B. D'Aguanno, and J. Goodwin, J. Chem. Phys. 95, 494 (1991).

[70] Y. Levin, M. C. Barbosa, and M. N. Tamashiro, Europhys. Lett. 41, 123 (1998).

[71] M. Morvan, D. Espinat, J. Lambard and Th. Zemb, Colloid Surf. A 82, 193 (1994).

[72] F. Pignon, A. Magnin, J.-M. Piau, B. Cabane, P. Lindner and O. Diat, Phys. Rev. E 56, 3281 (1997).

[73] J. M. Saunders, J. W. Goodwin, R. M. Richardson, and B. Vincent, J. Phys. Chem. B 103, 9211 (1999).

[74] P. Levitz, E. Lecolier, and A. Mourchid, Europhys. Lett. 49, 672 (2000).

[75] S. Bhatia, J. Barker, and A. Mourchid, Langmuir 19, 532 (2003).

[76] F. Schosseler, R. Skouri, J. P. Munch, and S. J. Candau, J. Phys. II France 4, 1221 (1994).

[77] D. P. Norwood, M. Benmouna, and W. F. Reed, Macromolecules 29, 4293 (1996).

[78] J. R. C. van der Maarel, W. Jesse, M. E. Kuil, and A. Lapp, Macromolecules 29, 2039 (1996).

[79] S. Förster, M. Schmidt, M. Antonietti, Polymer 31, 781 (1990).

[80] F. Boue, J. P. Cotton, A. Lapp, G. Jannink, J. Chem. Phys. 101, 2562 (1994).

[81] B. D. Ermi and E. J. Amis, Macromolecules 31, 7378 (1998).

[82] P. Linse and V. Lobaskin, Phys. Rev. Lett. 83, 4208 (1999).

[83] K. Kassapidou, W. Jesse, M. E. Kuil, A. Lapp, S. Egelhaaf, and J. R. C. van der Maarel, Macromolecules 30, 2671 (1997).

[84] E. E. Maier, S. F. Schulz, and R. Weber, Macromolecules 21, 1544 (1988).

[85] B. Ruzika, L. Zulian, and G. Ruocco, Phys. Rev. Lett. 93, 258301 (2004)

[86] R. Piazza, Curr. Opin. Colloid Interface Sci. 5, 38 (2000).

[87] G. Pellicane, D. Costa, and C. Caccamo, J. Phys.: Condens. Matter 15, 375 (2003).

[88] F. Sciortino, S. Mossa, E. Zaccarelli, and P. Tartaglia, Phys. Rev. Lett 93, 055701 (2004).

[89] D. Ruelle, Statistical Mechanics: Rigorous Results, (Imperial, London, 1969). 